\title{
Kinetic and Thermodynamic Analysis of Leech-Derived Tryptase Inhibitor Interaction with Bovine Tryptase and Bovine Trypsin
}

\author{
Fulvio Erba ${ }^{1}, *$, Laura Fiorucci ${ }^{1}$, Christian $\mathbf{P .}$ \\ Sommerhoff ${ }^{2}$, Massimiliano Coletta ${ }^{1}$ and \\ Franca Ascoli ${ }^{1}$ \\ ${ }^{1}$ Dipartimento di Medicina Sperimentale e \\ Scienze B iochimiche, U niversità Tor Vergata, via di Tor \\ Vergata 135, I-00133 Roma, Italy \\ ${ }^{2}$ Abteilung für Klinische Chemie und Klinische \\ Biochemie in der Chirurgischen Klinik und Poliklinik \\ Innenstadt der Ludwig-M aximilians-Universität, \\ Nussbaumstr. 20, D-80336 München, Germany \\ *Corresponding author
}

The interaction of leech-derived tryptase inhibitor (LDTI) with bovine liver capsule tryptase (BLCT) and bovine trypsin has been studied using both thermodynamic and kinetic approaches. Several differences were detected: (i) the equilibrium affinity of LDTI for BLCT $\left(K_{a}=8.9 \times 10^{5} \mathrm{M}^{-1}\right)$ is about 600-fold lower than that for bovine trypsin $\left(K_{a}=5.1 \times 10^{8} \mathrm{M}^{-1}\right)$; (ii) LDTI behaves as a purely non-competitive inhibitor of BLCT, while it is a purely competitive inhibitor of bovine trypsin. These functional data are compared with those previously reported for the LDTI binding to human tryptase, where tight inhibition occurs at two of the four active sites of the tetramer $\left(K_{a}=7.1 \times 10^{8} \mathrm{M}^{-1}\right)$. Amino acid sequence alignment of BLCT, human $\beta$ IItryptase and bovine trypsin allows us to infer some possible structural basis for the observed functional differences.

Key words: LDTI / Mast cell tryptase / P rotease inhibitor / Trypsin.

\section{Introduction}

Tryptases are the major trypsin-like proteases of mast cell secretory granules (Schwartz, 1990). They have been isolated from a variety of organisms, such as man (S mith et al., 1984; Cromlish et al., 1987; Harvima et al., 1988), rat (Kido et al., 1985; Braganza and Simmons, 1991), dog (Schechter et al., 1988), and cow (Fiorucci et al., 1992). Bovine liver capsule tryptase (BLCT) is an oligomer with an apparent $\mathrm{M}_{\mathrm{r}}$ of 360000 , composed of $35-38 \mathrm{kDa}$ subunits (Erba et al., 1998), displaying about 75\% identity in amino acid sequence with tryptases from other species (Pallaoro et al., 1996). Stability and enzymatic activity of BLCT, as well as of human tryptase, is affected by several factors including time, presence of glycerol, ionic strength and glycosaminoglycan interaction (Schechter et al., 1993, 1995; Addington and J ohnson, 1996; Erba et al., 1998).

Binding studies have shown that the Kunitz-type bovine pancreatic trypsin inhibitor (BPTI) is able to inhibit BLCT in vitro (Fiorucci et al., 1995a); co-localization of the inhibitor and the protease in the same compartment of bovine mast cells (Fiorucci et al., 1995b) has suggested a physiological significance for this interaction. Interestingly, endogenous inhibitors, able to modulate human tryptase activity, have not been found yet. So far the only natural inhibitor known for human tryptase is a small 46 amino acid protein, which has been isolated from the leech Hirudo medicinalis and named LDTI (leech-derived tryptase inhibitor; Sommerhoff et al., 1994). LDTI inhibits trypsin and chymotrypsin activity with a $K_{i}$ of $0.9 \mathrm{~nm}$ and $20 \mathrm{~nm}$, respectively, while inhibition of human tryptase occurs with a $K_{i}$ of $1.4 \mathrm{~nm}$ (Sommerhoff et al., 1994; Stubbs et al., 1997).

Here we report an investigation on the LDTI binding to both BLCT and bovine trypsin, using a steady-state kinetic approach to measure the equilibrium and kinetic properties of the interaction. The results obtained are compared with those reported previously for the inhibition of human tryptase by LDTI (Sommerhoff et al., 1994; Stubbs et al., 1997). On the basis of the amino acid homology, a possible structural basis is suggested to explain the functional differences between bovine and human tryptases.

\section{Results}

B inding experiments of LDTI to bovine trypsin (Figure 1A) yielded a value of $K_{a}=5.069( \pm 0.3) \times 10^{8} \mathrm{M}^{-1}$, in good agreement with the already reported values of $K_{i}=0.9$ $1.8 \mathrm{~nm}$ (Sommerhoff et al., 1994). Data points were fitted according to a linkage scheme (Wyman, 1964) and the following general equation was employed for the analysis of the trypsin-LDTI interaction:

$y=1-\left(K_{a} x /\left(1+K_{a} x\right)\right)$

Equation 1

where $y$ is the fraction of residual activity, $x$ is the concentration of free inhibitor and $\mathrm{K}_{\mathrm{a}}$ is the equilibrium association constant. The calculated curve is compatible with a simple competitive mechanism for trypsin inhibition by LDTI. This finding is in line with the crystal structure of the complex between LDTI and porcine trypsin (Di M arco and Priestle, 1997; Stubbs et al., 1997), which shows that the inhibitor binds to the active site of trypsin in a substratelike manner with residue $P 1$ (Lys 8 ) interacting with residue 


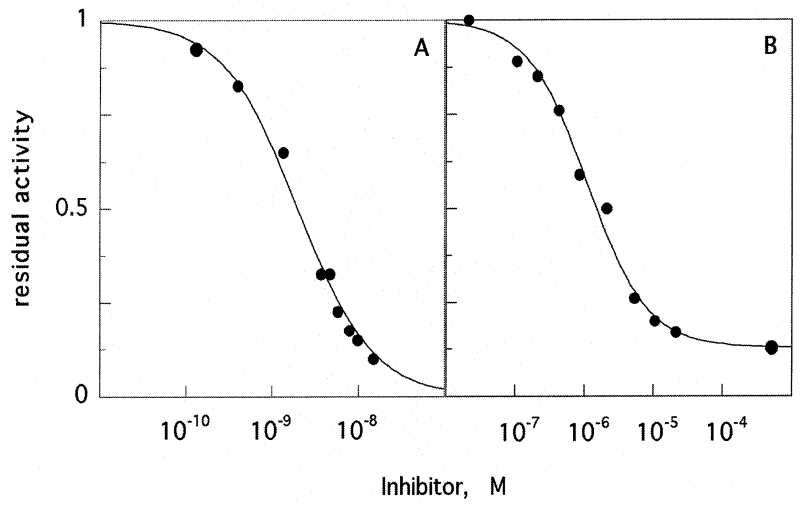

Fig. 1 Binding of LDTI to Bovine Trypsin (A) and Bovine Tryptase (B).

Bovine trypsin ( $0.5 \mathrm{~nm}$ active sites), or BLCT (2.5 nM active sites) were incubated in $0.1 \mathrm{M} \mathrm{Tris}-\mathrm{HCl} \mathrm{pH} 8.0$, at $30^{\circ} \mathrm{C}$ for 30 min with increasing amounts of LDTI. Residual enzymatic activity was assayed using Boc-Phe-Ser-Arg-MCA as a substrate. The continuous lines in panels $(A)$ and $(B)$ represent the best fit of data using Eq. 1 and Eq. 2, respectively.

Asp 189 in the primary specificity pocket of the enzyme.

On the other hand, LDTI binding to BLCT (Figure 1B) displays markedly different features, suggesting a different interaction mechanism, which was analyzed with a modified formula, namely

$y=1-\left(K_{a} x /\left(1+K_{a} x\right)\right) Q$

Equation 2

where all parameters have the same meaning as in Eq. 1 , and $Q$ represents the maximum inhibition obtained. The best fits were calculated with a non-linear regression analysis using a $M$ arquardt algorithm.

The binding constant $\mathrm{K}_{\mathrm{a}}=8.9( \pm 3.2) \times 10^{5} \mathrm{M}^{-1}$ is about three orders of magnitude lower than that found for trypsin, indicating a low stability of the BLCT-LDTI complex. The most striking difference compared to the trypsin-LDTI interaction is given by the finding that, even at high inhibitor concentration, the BLCT-LDTI binding curve displays an asymptotic residual activity, amounting to about $13 \%$ (corresponding to $\mathrm{Q}=0.87$ in Eq. 2), as if a certain percentage of the enzyme is never inhibited by LDTI. The presence of other contaminating serine proteases can be ruled out, since only two $\left[{ }^{3} \mathrm{H}\right]$-DFP labeled bands corresponding to tryptase were detected on SDS gels. Thus, the behavior shown in Figure 1B can be accounted for by the interaction of LDTI at a binding site different from the primary specificity pocket, decreasing the catalytic rate constant $k_{\text {cat }}$ according to a non-competitive inhibitory mechanism.

In order to test this possibility, we carried out a steadystate kinetic investigation at different substrate and inhibitor concentrations (Figure 2). The results obtained indeed suggest a simple non-competitive inhibitory mechanism because the data can be described by an inhibitor-independent $\mathrm{K}_{\mathrm{m}}$ of $4.3 \times 10^{-5} \mathrm{M}^{-1}$ and an inhibitordependent $\mathrm{k}_{\mathrm{cat}}$ according to the formula

${ }^{\mathrm{obs}} \mathrm{k}_{\text {cat }}={ }^{0} \mathrm{k}_{\text {cat }}\left(1-\left(\mathrm{K}_{\mathrm{a}}[\mathrm{l}] /\left(1+\mathrm{K}_{\mathrm{a}}[\mathrm{l}]\right)\right) \mathrm{Q}\right)$

Equation 3

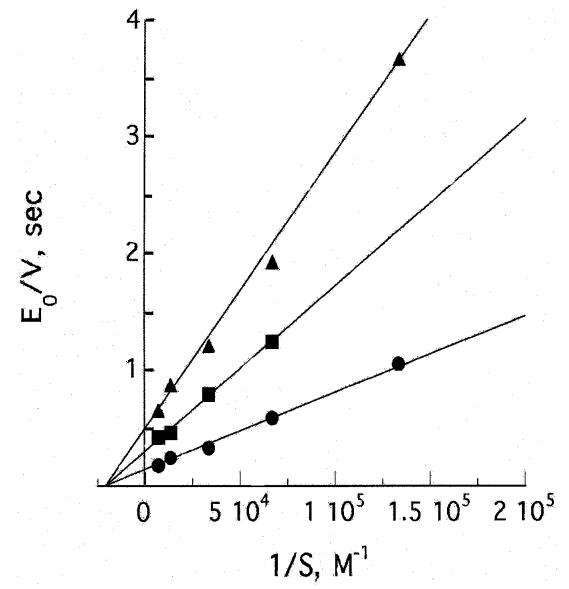

Fig. 2 Lineweaver-Burk Plots of Bovine Tryptase (5 nм Active Sites) in the Absence $(\bullet)$ and in the Presence of $4.2 \mu \mathrm{M}(\mathbf{\square})$ and $8.4 \mu \mathrm{M} \operatorname{LDTI}(\mathbf{\Lambda})$.

The mixtures were incubated for $30 \mathrm{~min}$ at $30^{\circ} \mathrm{C}$ in $0.1 \mathrm{M}$ Tris- $\mathrm{HCl}$ $\mathrm{pH}$ 8.0. The residual proteolytic activity of each incubation mixture was then measured in the presence of different concentrations of BoC-Phe-Ser-Arg-MCA as a substrate.

Table 1 Catalytic and LDTI Binding Parameters of Bovine Trypsin and Bovine Tryptase in $0.1 \mathrm{M}$ Tris- $\mathrm{HCl}, \mathrm{pH} 8.0$, at $30^{\circ} \mathrm{C}$.

\begin{tabular}{|c|c|c|}
\hline & Bovine trypsin & BLCT \\
\hline \multicolumn{3}{|c|}{ Catalytic parameters } \\
\hline $\mathrm{k}_{\text {cat }}\left(\mathrm{s}^{-1}\right)$ & $14.7 \pm 1.2$ & $7.0 \pm 0.9$ \\
\hline $\mathrm{K}_{\mathrm{m}}(\mathrm{M})$ & $3.05( \pm 0.4) \times 10^{-5}$ & $4.0( \pm 1.0) \times 10^{-5}$ \\
\hline \multicolumn{3}{|c|}{ LDTI binding parameters } \\
\hline$K_{a}\left(M^{-1}\right)$ & $5.08( \pm 0.24) \times 10^{8}$ & $8.9( \pm 3.2) \times 10^{5}$ \\
\hline$k_{o n}\left(M^{-1} s^{-1}\right)$ & $3.3( \pm 0.4) \times 10^{5}$ & $4.2( \pm 1.3) \times 10^{3}$ \\
\hline $\mathrm{k}_{\text {off }}\left(\mathrm{s}^{-1}\right)$ & $6.6( \pm 1.9) \times 10^{-4}$ & $4.7( \pm 1.5) \times 10^{-3}$ \\
\hline
\end{tabular}

where ${ }^{\text {obs }} \mathrm{k}_{\text {cat }}$ is the observed catalytic rate constant, ${ }^{0} \mathrm{k}_{\text {cat }}$ $\left(=7.0 \mathrm{~s}^{-1}\right.$, see Table 1$)$ is the catalytic rate constant in the absence of LDTI, $K_{a}\left(8.9 \times 10^{5} \mathrm{M}^{-1}\right)$ and $\mathrm{Q}(0.87)$ have the same meaning as in Eq. 2.

The different interaction mechanism of LDTI with bovine trypsin and BLCT was further investigated by studying the kinetic aspects of the interaction of LDTI with the two enzymes. Figure $3 \mathrm{~A}$ shows the progress curves obtained forbovine trypsin, applying Scheme 1 for a purely competitive inhibition, to fit the kinetic parameters:

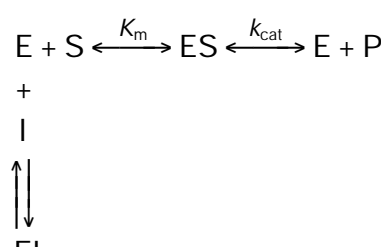

Scheme 1

EI

where $\mathrm{K}_{\mathrm{m}}$ is the Michaelis-Menten constant, $\mathrm{K}_{\mathrm{a}}$ is the inhibitor association binding constant to the free enzyme and $\mathrm{k}_{\text {cat }}$ is the catalytic rate constant. The continuous lines ofFigure $3 \mathrm{~A}$ were obtained with the same set of kinetic rate constants, the various curves referring to different LDTI 
concentrations; the only constraint imposed on the values of rate constants was that $k_{\text {on }} / k_{\text {off }}=K_{a}$. It is evident that Scheme 1 is appropriate for describing the kinetics of LDTI

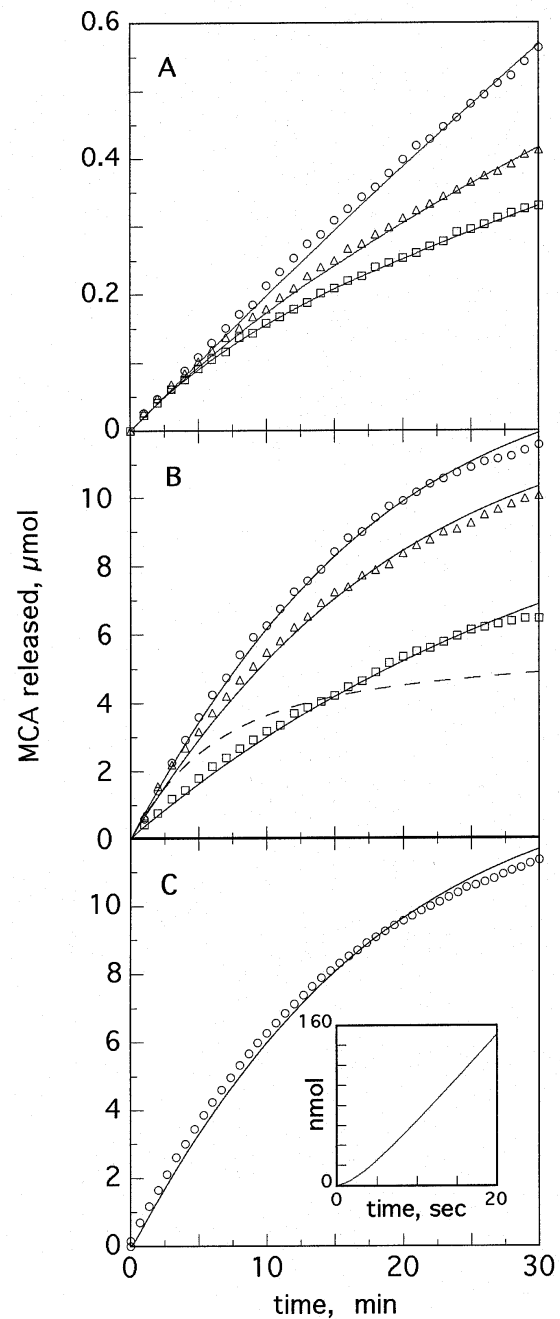

Fig. 3 Kinetics of the Interaction of LDTI with B ovine Trypsin and BLCT.

(A) Bovine trypsin-LDTI association. Enzymatic activity of bovine trypsin (0.5 nM active sites) toward Boc-Phe-Ser-Arg-MCA in $0.1 \mathrm{M}$ Tris- $\mathrm{HCl} \mathrm{pH} 8.0$, at $30^{\circ} \mathrm{C}$, was recorded for $40 \mathrm{~min}$ in the absence $(O)$ or in the presence of $1.65 \mathrm{~nm}(\triangle)$ and $4.26 \mathrm{~nm}(\square)$ LDTI. Continuous lines represent the best fit of data using an equation based on a competitive mechanism of inhibition (see Scheme 1) (B) BLCT-LDTI association. Enzymatic activity of BLCT (2.5 nM active sites) was recorded under the same conditions, either in the absence $(O)$ or in the presence of $2 \mu \mathrm{M}(\triangle)$ and $16 \mu \mathrm{M}(\square)$ LDTI. Continuous lines represent the best fit of data using an equation based on a noncompetitive mechanism of inhibition (see Scheme 2). The dashed line represents the fitting of the progress curve obtained at the highest LDTI concentration, according to Scheme 1 (competitive inhibition mechanism). (C) BLCT-LDTI complex dissociation. BLCT ( $50 \mathrm{~nm}$ active sites) was incubated with $2 \mu \mathrm{M}$ LDT in $200 \mu \mathrm{l}$ of $0.1 \mathrm{M}$ Tris- $\mathrm{HCl}, \mathrm{pH} 8.0$, for $30 \mathrm{~min}$ at $30^{\circ} \mathrm{C}$. BLCT-LDTI complex dissociation was then measured by monitoring enzymatic activity for 30 min after 10 -fold dilution with the same buffer (O). The continuous line represents the theoretical progress curve of the equilibrium relaxation calculated with the rate constants deriving from the fitting of the BLCT-LDTI association curve. The onset of the theoretical progress curve is shown in the inset. binding to trypsin, confirming that the interaction follows a simple competitive mechanism.

Figure 3B displays the progress curves obtained with $B L C T$ at different concentrations of LDTI. The dashed line represents the best fit according to Scheme 1 obtained at the highest inhibitor concentration used $(16 \mu \mathrm{M})$; it is obvious that a simple competitive mechanism does not properly describe the dynamic behavior of LDTI binding to BLCT. We have then attempted a description of the system according to Scheme 2:

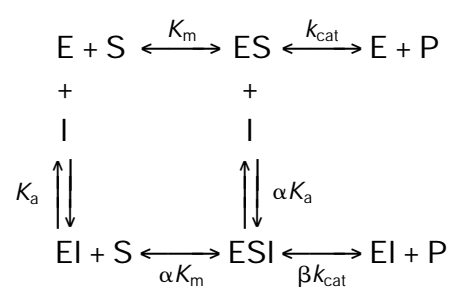

Scheme 2

considering a purely noncompetitive mechanism, where $\mathrm{K}_{\mathrm{a}}, \mathrm{K}_{\mathrm{m}}$ and $\mathrm{K}_{\mathrm{cat}}$ have the same meaning as in Scheme 1. $\alpha$ is the interaction factor between the binding of inhibitor (or substrate) to the free enzyme and that to the substratebound (or inhibitor-bound) enzyme, with $\alpha>1$ for a positive interaction, $\alpha<1$ for a negative interaction and $\alpha=1$ for a purely noncompetitive mechanism. $\beta$ is the interaction factor for the rate of substrate catalysis between the ES complex and the ESI complex, with $\beta>1$ if the ESI form is catalyzed more quickly than $E S$, and $\beta<1$ if the opposite is observed. Data points were analyzed setting $\alpha=1$ (i. e., the inhibitor does not affect the intrinsic affinity of substrate for the enzyme and vice versa) and $\beta=0.13$ (corresponding to the ratio of $k_{\text {cat }}$ for ESI and ES species, see Scheme 2). The continuous lines in Figure 3B correspond to such a mechanism. The various curves employ the same set of rate constants and refer to different LDTI concentrations, with the same constraint imposed for LDTI binding to trypsin (i. e. $\mathrm{k}_{\text {on }} / \mathrm{K}_{\text {off }}=\mathrm{K}_{\mathrm{a}}$ ). Scheme 2 indeed appears considerably more appropriate for a quantitative description of the BLCT-LDTI binding.

An important aspect of the applicability of the scheme concerns the reliability of kinetic parameters reported in Table 1. Thus, a too slow dissociation rate constant for the BLCT-LDTI complex (i. e. $<10^{-5} \mathrm{~s}^{-1}$ ) might impair the validity of data reported in Figure 2 , since in such a case reequilibration after incubation would be too slow to be complete during the steady-state observations. Therefore, we carried out a direct measurement of the complex dissociation process, incubating the enzyme and the inhibitor under conditions in which about $70 \%$ enzyme inhibition occurs. 10-fold dilution of this incubation mixture into the assay buffer containing the substrate induces a shift toward a new equilibrium by dissociation of the inhibitor from the enzyme. The substrate cleavage, monitored as a function of time, corresponds to the progressive dissociation of the inhibitor/enzyme complex. Figure $3 \mathrm{C}$ shows data obtained from this experimental approach and the continuous line is the expected behavior of the system according to the parameters reported in Table 1. It is immedi- 
ately evident that the rate constants are appropriate for a quantitative description of the inhibitor association and dissociation processes, further reinforcing the interpretation according to Scheme 2 . It must be noted that the shape of the progress curve reported in Figure $3 \mathrm{C}$ does not show the expected upward curvature corresponding to the increase of enzyme activity following inhibitor dissociation. The inconsistency is only apparent since such an increase occurs in the very early stages of the process (within the first $20 \mathrm{~s}$ ), confirming the relatively fast dissociation constant (see Figure $3 \mathrm{C}$ inset). The upward curvature occurs at a time interval much shorter than the actual half-time of the inhibitor dissociation process (about $2 \mathrm{~min}$ according to the $\mathrm{k}_{\text {off }}$ value reported in Table 1 ), simply because the difference in the rate for substrate catalysis, before and after the inhibitor dissociation, is such that the inhibitor-free species dominates the apparent rate of substrate catalysis. Thus the shift in equilibrium occurs in a relatively short time ensuring that the observation time of the steady-state measurements is sufficient to guarantee the establishment of the equilibrium.

\section{Discussion}

A comparison between the kinetic and thermodynamic parameters obtained for bovine trypsin and BLCT (Table 1) indicates that the $\sim 600$-fold different affinity of LDTI for the two enzymes is mostly due to the kinetic association process ( $k_{\text {on }}$ values differ $\approx 80$-fold) suggesting the presence of an energetic barrier (amounting to $\Delta \Delta \mathrm{G}^{\neq} \sim 11 \mathrm{~kJ} /$ mol) hind ering the formation of the enzyme-inhibitor complex. A much smaller difference is observed for the complex dissociation, which is only 7-fold faster in BLCT than in trypsin, indicating that once the El complex is formed its stability is not drastically different between the two enzymes. A structural interpretation of this difference is not straightforward, also in view of the fact that the different inhibition mechanism for the two enzymes may imply a somewhat different enzyme-inhibitor interaction site. However, independently of this uncertainty, the decreased association rate and the faster dissociation rate constant of LDTI observed in BLCT with respect to bovine trypsin (see Table 1) suggests a weaker interaction of the LDTI P1 residue with the primary specificity pocket in bovine tryptase (Erba et al., 1998). Such a feature reflects the Asp189 replacement by Asn189 in BLCT (Figure 4), which results in a decreased negative charge at the bottom of the pocket with respect to that of bovine trypsin and human $\beta$ Il-tryptase. This difference might account for the much higher affinity of LDTI for human $\beta$ Il-tryptase with respect to BLCT. However, at nanomolar concentrations LDTI induces only $50 \%$ inhibition of the human tryptase tetramer (Sommerhoff et al., 1994). Other structural differences between bovine and human $\beta$ II-tryptase may be considered. Thus, modeling of the LDTI-human tryptase complex, as well as inhibition experiments of human $\beta$ II-tryptase with recombinant LDTI variants (Stubbs et al., 1997), indicate that the complex formation is favored by the juxtaposition of Lys1-Lys2 at the N-terminus of LDTI with residues Asp143 and Asp147 of the 147 loop of the tryptase monomer where LDTI is bound (Pereira et al., 1998; Sommerhoff et al., 1999). This interaction is impaired in BLCT, since the 147 loop lacks Asp143 and Asp147, which are substituted by Asn and Gly, respectively (Figure 4). Another relevant structural difference between human and bovine tryptase is the substitution of Arg173 with a Pro residue in bovine tryptase (Figure 4). This residue, located at the end of the 173 flap, may force this structure to assume a conformation resulting in an active site more accessible to macromolecular inhibitors such as LDTI or BPTI (Fiorucci et al., 1995a). It is noteworthy that rat tryptase shows a Pro residue in the same position and is inhibited by macromolecular inhibitors (Braganza and

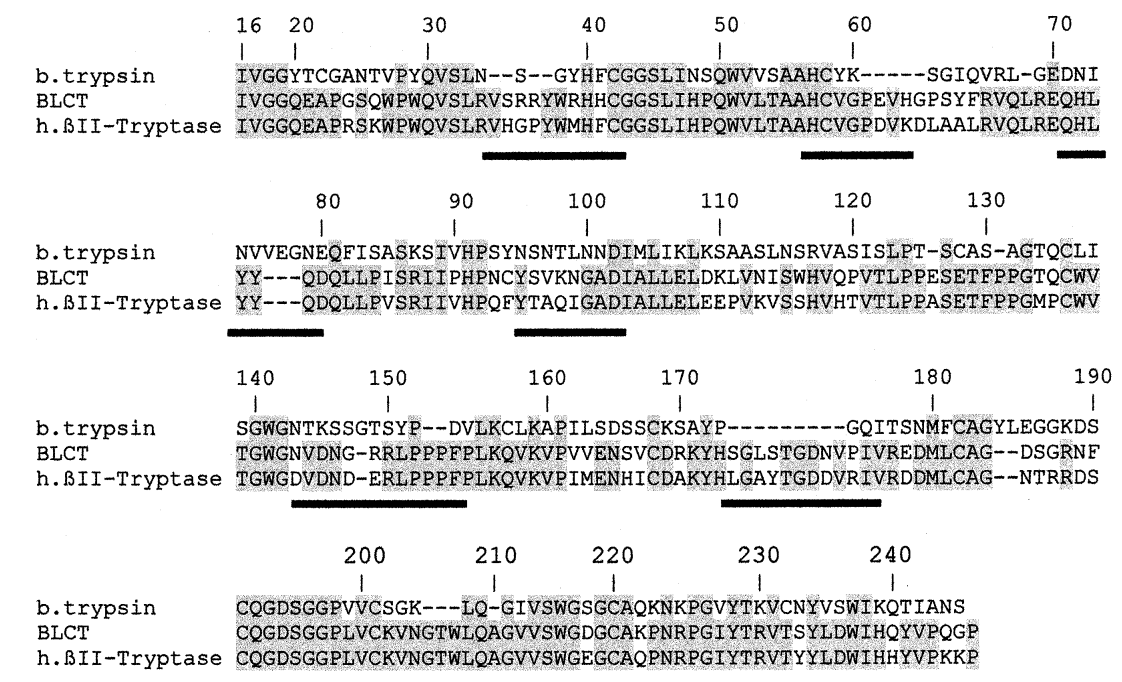

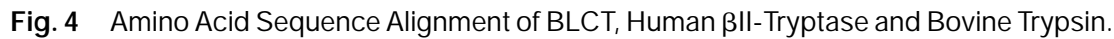

Multiple sequence alignment was performed using the ClustalW program. Identical amino acids are shaded. The six surface loops involved in the contacts between monomers in human Bll-tryptase (Pereira et al., 1998) are underlined. Numbering refers to the chymotrypsinogen sequence. 
Simmons, 1991), similarly to BLCT. Moreover, many sequence changes occur at the six surface loops (Figure 4), which, in the human $\beta$ II-tryptase tetramer, are involved in the two different kinds of contacts between subunits (Pereira et al., 1998). Changes in the loop sequence and length were pointed out recently for human and mouse TMT (transmembrane tryptases) with respect to human $\beta l$-tryptase, and it was suggested that it is unlikely that these new members of the tryptase family form a similar tetrameric structure as in human $\beta$ Il-tryptase (Wong et al., 1999). Likewise, a different pattern of interaction among monomers could occur in BLCT, with a possible change in the central oval pore geometry, which could become more accessible to macromolecular inhibitors.

Finally, is worth remembering that the endogenous tryptase inhibitor (BPTI) is contained in bovine mast cells (Fiorucci et al., 1995b), and this may have some peculiar influence on the role and on the functional properties of bovine tryptase. Therefore, it appears likely that tryptases from different species differ more than expected in respect to activity, regulation, and inhibition, and that this may be related to different metabolic aspects in the various species.

\section{Materials and Methods}

BLCT was isolated and purified as previously described (Fiorucci etal., 1992). The fresh stock solution (2 - $4 \mu \mathrm{M}$ active sites in $10 \mathrm{~mm}$ MES, $2 \mathrm{M} \mathrm{NaCl}$, pH 6.1) was titrated and used directly for the inhibition experiments. The enzyme preparation was labeled with $\left[{ }^{3} \mathrm{H}\right]$-diisopropyl fluoro-phosphate and subjected to SDS-PAGE. Autoradiography of the gel showed only the doublet band corresponding to tryptase. rLDTI was obtained and purified as previously reported (Auerswald et al., 1994; Pohlig et al., 1996). Bovine trypsin (type XIII) and the fluorogenic substrate B oc-P he-Ser-ArgMCA were purchased from Sigma-Aldrich. All other chemical products were of analytical grade.

\section{Equilibrium Binding Experiments}

Steady-state experiments were performed in $0.1 \mathrm{M}$ Tris- $\mathrm{HCl}, \mathrm{pH}$ 8.0 (assay buffer) at $30^{\circ} \mathrm{C}$. BLCT $(2.5 \mathrm{~nm}$ active sites) or bovine trypsin ( $0.5 \mathrm{~nm}$ active sites) were incubated for $30 \mathrm{~min}$ with different amounts of LDTI and then assayed for proteolytic activity using Boc-Phe-Ser-Arg-MCA as a substrate $(14 \mu \mathrm{M})$. The fluorescence of 7-amino-4-methyl-coumarin (MCA) released from the substrate was monitored in a thermostated spectrofluorimeter Kontron SFM 25 (excitation at $370 \mathrm{~nm}$, emission at $460 \mathrm{~nm}$; Pallaoro et al., 1996). The signal was recorded for at least five minutes. A plot of residual activity versus free inhibitor concentration was generated.

For the determination of the inhibition mechanism, $\mathrm{K}_{\mathrm{m}}$ and $\mathrm{V}_{\max }$ values for the hydrolysis of Boc-Phe-Ser-Arg-MCA were obtained by measuring tryptase activity in the assay buffer at $30^{\circ} \mathrm{C}$ at different substrate concentrations (i. e., $1-70 \mu \mathrm{M}$ ), in the presence of two concentrations of LDTI (i. e. 4.2 and $8 \mu \mathrm{M}$ ), following 30 min pre-incubations. Data were processed into LineweaverBurk plots.

\section{Kinetic Experiments}

Kinetic measurements were performed in the assay buffer at $30^{\circ} \mathrm{C}$, by rapidly mixing the enzyme $(5.0 \mathrm{~nm}$ BLCT or $0.5 \mathrm{~nm}$ trypsin), Boc-Phe-Ser-Arg-MCA (14 $\mu \mathrm{M})$ and LDTI at different concentrations. Proteolytic activity was assayed as described above in the spectrofluorimeter. The progress curves were recorded for at least $30 \mathrm{~min}$. Data points were plotted in terms of product release as a function of time, and analyzed according to Scheme 1 (for a purely competitive inhibition) and Scheme 2 (for a noncompetitive inhibition) as described in the Results section. $\mathrm{Ki}$ netic parameters were obtained by an iterative least-squares fitting procedure employing our own program running under the MATLAB environment (The Student Edition of MATLAB, 1992). A set of differential equations was used for each species represented in the proposed scheme(s) according to the Runge-Kutta method (Smith, 1978). At each step the concentrations of all molecular species, including free substrate, free enzyme and free inhibitor were recalculated and updated. For fast equilibria, such as the process concerning $\mathrm{K}_{\mathrm{m}}$, we considered the ratio [ES]/[E] [S] constant over the time range of the phenomena observed, therefore no specific experimental conditions are required to describe the kinetic progress curves and to determine kinetic parameters.

The kinetic dissociation constant for the BLCT-LDTI complex was evaluated by incubating BLCT ( $50 \mathrm{~nm}$ active sites) and LDTI $(2 \mu \mathrm{M})$ in $200 \mu$ l of the assay buffer for $30 \mathrm{~min}$ at $30^{\circ} \mathrm{C}$, a condition that ensures a $70 \%$ enzyme inhibition (see Figure 1B). $1.8 \mathrm{ml}$ of the same buffer containing $14 \mu \mathrm{M}$ Boc-Phe-Ser-Arg-MCA were then added. The new concentration condition induces a shift toward a new equilibrium in which no more than $20 \%$ enzyme inhibition occurs. The fluorescence signal recorded for 30 min after mixing corresponds to the enzyme activity, which increases following LDTI dissociation and can be simulated employing the same Runge-Kutta program utilized for the association kinetic constants (see above).

Standard deviations were derived from several experiments (at least three for each experimental condition).

\section{Acknowledgements}

This investigation was supported by MURST-PRIN 1997, by MURST-CNR Biotechnology Program L.95/95, Italy, and by the Sonderforschungsbereich 469 of the University of Munich, Germany.

\section{References}

Addington, A.K., and J ohnson, D.A. (1996). Inactivation of human lung tryptase: evidence for a re-activatable tetrameric intermediate and active monomers. B iochemistry 35, 13511 - 13518.

Auerswald, E.A., Morenweiser, R., Sommerhoff, C.P., P iechottka, G.I., Eckerskorn, C., Gurtler, L.G., and Fritz, H. (1994). Recombinant leech-derived tryptase inhibitor: construction, production, protein chemical characterization and inhibition of HIV-1 replication. Biol. Chem. Hoppe-Seyler 375, 695- 703.

Braganza, V.J ., and Simmons, W.H. (1991). Tryptase from rat skin: purification and properties. Biochemistry 30, 4997- 5007.

Cromlish, J.A., Seidah, N.G., Marcinkiewicz, M., Hamelin, J ., Johnson, D.A., and Chretien, M. (1987). Human pituitary tryptase: molecular forms, NH2-terminal sequence, immunocytochemical localization, and specificity with prohormone and fluorogenic substrates. J. Biol. Chem. 262, 1363- 1373.

Di Marco, S., and Priestle, J.P. (1997). Structure of the complex of leech-derived tryptase inhibitor (LDTI) with trypsin and modeling of the LDTI-tryptase system. Structure 11, 1465 - 1474. 
Erba, F., Fiorucci, L., Coletta, M., and Ascoli, F. (1998). Bovine mast cell tryptase inactivation: effect of temperature. Peptides $19,437-443$.

Fiorucci, L., Erba, F., and Ascoli, F. (1992). Bovine tryptase: purification and characterization. Biol. Chem. Hoppe-Seyler 373, $483-490$.

Fiorucci, L., Erba, F., Coletta, M., and Ascoli, F. (1995a). Evidence for multiple interacting binding sites in bovine tryptase. FEBS Lett. 363, $81-84$.

Fiorucci, L., Erba, F., Falasca, L., Dini, L., and Ascoli, F. (1995b). Localization and interaction of bovine pancreatic trypsin inhibitor and tryptase in the granules of bovine mast cells. Biochim. Biophys. Acta 1243, $407-413$.

Harvima, I.T., Schechter, N., Harvima, R., and Fraki, J .E. (1988) Human skin tryptase: purification, partial characterization and comparison with human lung tryptase. Biochim. Biophys. Acta 957, 71- 80.

Kido, H., Fukusen, N., and Katunuma, N. (1985). Chymotrypsinand trypsin-type serine proteases in rat mast cells: properties and functions. Arch. Biochem. Biophys. 239, 436 - 443.

Pallaoro, M., Gambacurta, A., Fiorucci, L., Mignogna, G., Barra, D., and Ascoli, F. (1996). CDNA cloning and primary structure of tryptase from bovine mast cells, and evidence for the expression of bovine pancreatic trypsin inhibitor mRNA in the same cells. Eur. J . Biochem. 237, $100-105$.

Pereira, P.J.B., Bergner, A., Macedo-Ribeiro, S., Huber, R., Matschiner, G., Fritz, H., Sommerhoff, C.P., and Bode, W. (1998). Human beta-tryptase is a ring-like tetramer with active sites facing a central pore. Nature 392, 306-311.

Pohlig, G., Fendrich, G., Knecht, R., Eder, B., Piechottka, G., Sommerhoff, C.P., and Heim, J . (1996). Purification, characterization and biological evaluation of recombinant leech-derived tryptase inhibitor (rLDTI) expressed at high level in the yeast Saccharomyces cerevisiae. Eur. J . Biochem. 241, 619-626.

Schechter, N.M., Slavin, D., Fetter, R.D., Lazarus, G.S., and Fraki, J.E. (1988). Purification and identification of two serine class proteinases from dog mast biochemically and immunologically similar to human proteinases tryptase and chymase. Arch. Biochem. Biophys. 262, $232-244$.

Schechter, N.M., Eng, G.Y., and McCaslin, D.R. (1993). Human skin tryptase: kinetic characterization of its spontaneous inactivation. Biochemistry 32, 2617-2625.
Schechter, N.M., Eng, G.Y., Selwood, T., and McCaslin, D.R. (1995). Structural changes associated with the spontaneous inactivation of the serine proteinase human tryptase. Biochemistry $34,10628-10638$

Schwartz, L.B. (1990). Tryptase from human mastcells: Biochemistry, B iology and clinical Utility. In: Neutral Proteases of Mast Cells, L.B. Schwartz, ed. (Basel, Switzerland: Karger), pp. 90 113.

Smith, G. D. (1978). Numerical solution of partial differential equations, $2^{\text {nd }}$ Edition (Oxford, UK: Clarendon Press).

Smith, T.J ., Houghland, M.W., and J ohnson, D.A. (1984). Human lung tryptase. Purification and characterization. J . Biol. Chem. 259, 11046 - 11051

Sommerhoff, C.P., Soellner, C., Mentele, R., Piechottka, G.P., Auerswald, E.A., and Fritz, H.A. (1994). A Kazal-type inhibitor of human mast cell tryptase: isolation from the medical leech Hirudo medicinalis, characterization, and sequence analysis. Biol. Chem. Hoppe-Seyler 375, 685- 694.

Sommerhoff, C.P., Bode, W., Pereira, P.J ., Stubbs, M.T., Stürzebecher, J., Piechottka, G.P., Matschiner, G., and Bergner, A. (1999). The structure of the human betall-tryptase tetramer: fo(u)r better or worse. Proc. Natl. Acad. Sci. USA 96, 10984 10991.

Stubbs, M.T., Morenweiser, R., Stürzebecher, J ., Bauer, M., Bode, W., Huber, R., Piechottka, G.P., Matschiner, G., Sommerhoff, C. P., Fritz, H., and Auerswald, E.A. (1997). The three-dimensional structure of recombinant leech-derived tryptase inhibitor in complex with trypsin. Implications for the structure of human mast cell tryptase and its inhibition. J . Biol. Chem. 272, 19931 19937.

The Student Edition of MATLAB (1992). The MathWorks, Inc.

Wong, G.W., Tang, Y., Feyfant, E., Sali, A., Li, L., Li, Y., Huang, C., Friend, D.S., Krilis, S.A., Stevens, R.L. (1999). Identification of a new member of the tryptase family of mouse and human mast cell proteases which possesses a novel $\mathrm{COOH}$-terminal hydrophobic extension. J. Biol. Chem. 274, 30784- 30793.

Wyman, J . (1964). Facilitated diffusion and the possible role of myoglobin as a transport mechanism. Adv. Protein Chem. 19, 223-289.

Received April 26, 2000; accepted August 8, 2000 